\title{
Magnetic Properties of a Two-Dimensional Mixed-Spin System
}

\author{
J.-H. Park ${ }^{\mathrm{a}, 1}$, J. T. Culp ${ }^{\text {b }}$, D. W. Hall ${ }^{\text {, }}$ D. R. Talham ${ }^{\text {b }}$, M. W. Meisel ${ }^{\text {a }}$ \\ ${ }^{\mathrm{a}}$ Department of Physics and National High Magnetic Field Laboratory, University of Florida, Gainesville, FL 32611-8440, USA. \\ ${ }^{\mathrm{b}}$ Department of Chemistry, University of Florida, Gainesville, FL 32611-7200, USA. \\ ${ }^{\mathrm{c}}$ National High Magnetic Field Laboratory, Florida State University, Tallahassee, FL 32310, USA.
}

\begin{abstract}
Using a Langmuir-Blodgett (LB) synthesis method, novel two-dimensional (2D) mixed-spin magnetic systems, in which each magnetic layer is both structurally and magnetically isolated, have been generated. Specifically, a $2 \mathrm{D}$ Fe-Ni cyanide-bridged network with a face-centered square grid structure has been magnetically and structurally characterized. The results indicate the presence of ferromagnetic exchange interactions between the $\mathrm{Fe}^{3+}(S=1 / 2)$ and $\mathrm{Ni}^{2+}(S=1)$ centers.
\end{abstract}

Key words: two-dimensional magnetism; mixed-spin; Langmuir-Boldgett film

\section{Introduction}

Part of the interest in molecule-based magnets arises from the potential for forming low-dimensional structures with anisotropic physical properties. In this paper, we report on the magnetic behavior of quasi-twodimensional (2D) Fe-CN-Ni grid networks, in which each magnetic layer is both structurally and magnetically isolated. Culp et al. have developed a unique Langmuir-Blodgett (LB) synthesis method to transfer monolayers of a 2D Fe-CN-Ni grid network, formed at the air-water interface, as thin films onto various substrates [1]. This technique generates a new lowdimensional network which cannot be formed from homogeneous reaction conditions.

The structure of the resulting assemblies of mixedspin Fe-CN-Ni bilayers transferred as thin films onto Mylar is shown schematically in Fig. 1. Each deposition cycle produces a bilayer of the inorganic grid network sandwiched between an organic layer. The LB films have been structurally characterized by x-ray photoelectron spectroscopy (XPS), FT-IR spectroscopy, xray absorption fine structure (XAFS), and grazing in-

\footnotetext{
1 Corresponding author. E-mail: juhyun@phys.ufl.edu
}

cidence synchrotron x-ray diffraction (GIXD). These probes have allowed for the identification of a facecentered square grid structure with an $\mathrm{Fe}^{3+}-\mathrm{Ni}^{2+}$ separation of $5.1 \AA$, and an average structural domain size of $3600 \AA^{2}$ [1]. Adjacent bilayers are separated by $\approx 50 \AA$, and are effectively isolated from each other.

The existence of ferromagnetic exchange between the low spin $\mathrm{Fe}^{3+}(S=1 / 2)$ and $\mathrm{Ni}^{2+}(S=1)$ ions is expected after consideration of the exchange interaction in other cyanide bridged $\mathrm{Fe}^{3+}-\mathrm{Ni}^{2+}$ systems $[2,3]$. The ferromagnetic behavior in these and other metal cyanides has been rationalized by considering the

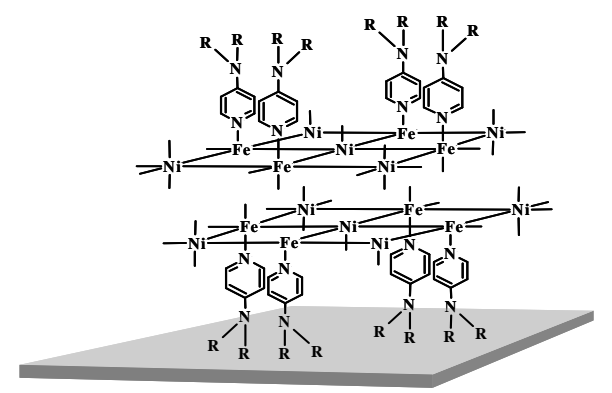

Fig. 1. The assembly of the Fe-CN-Ni grid bilayer transferred onto a thin mylar film. 


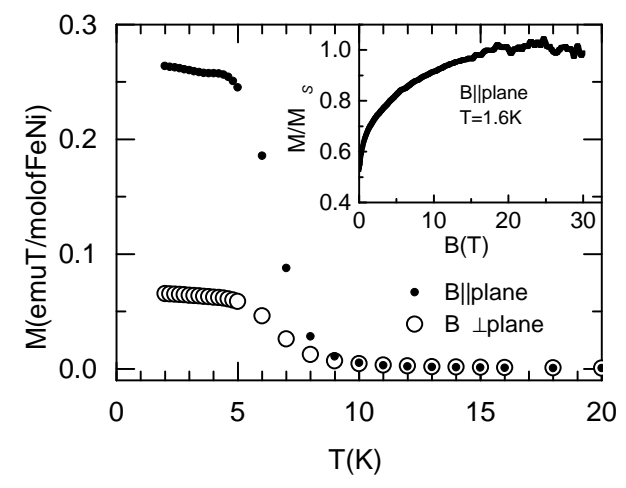

Fig. 2. Field cooled (FC) magnetization measured in $2 \mathrm{mT}$. The high field magnetization data at $1.6 \mathrm{~K}$ are shown in the inset.

d-orbital symmetries for the unpaired electrons in the respective metal centers. The magnetic orbital on the $\mathrm{Fe}^{3+}$ center is of $\mathrm{t}_{2 g}$ symmetry while the two $\mathrm{Ni}^{2+}$ spins are in orbitals of $e_{g}$ symmetry. The different symmetry orbitals do not overlap via the cyanide ligand, thereby resulting in the parallel alignment of the spins according to Hund's rule [4].

\section{Magnetic Measurements}

The magnetic properties of a sample containing 150 bilayers of the Fe-CN-Ni network on each side of a Mylar film $\left(10 \mathrm{~cm}^{2}\right)$ were investigated using SQUID magnetometry. Measurements were performed with the applied magnetic field oriented either parallel or perpendicular to the plane of the film. The field cooled (FC) magnetization versus temperature for both orientations, measured in a field of $2 \mathrm{mT}$, are shown in Fig. 2. Both orientations show a break at a $\mathrm{T}_{c}$ of $10 \mathrm{~K}$, and the saturated behavior below $5 \mathrm{~K}$ indicates the presence of magnetically ordered domains. The presence of longrange magnetic order at $2 \mathrm{~K}$ was verified by the measurements shown in Fig. 3. The material shows clear hysteresis loops with coercive fields of $14 \mathrm{mT}$ (parallel orientation) and $11 \mathrm{mT}$ (perpendicular orientation).

The assignment of a ferromagnetic state was made based on high field magnetization studies performed at the National High Magnetic Field Laboratory, Tallahassee (NHMFL). The magnetization of the film was measured up to $30 \mathrm{~T}$ using a vibrating sample magnetometer (VSM). As shown in Fig. 2 (inset), the magnetization of the sample saturates around $20 \mathrm{~T}$ without evidence for a spin-flop transition. The increased noise above $15 \mathrm{~T}$ is due to the addition of a second dc power supply generating the magnetic field. The absence of a spin flop discriminates against antiferromagnetic coupling and the presence of a ferrimagnetic state.

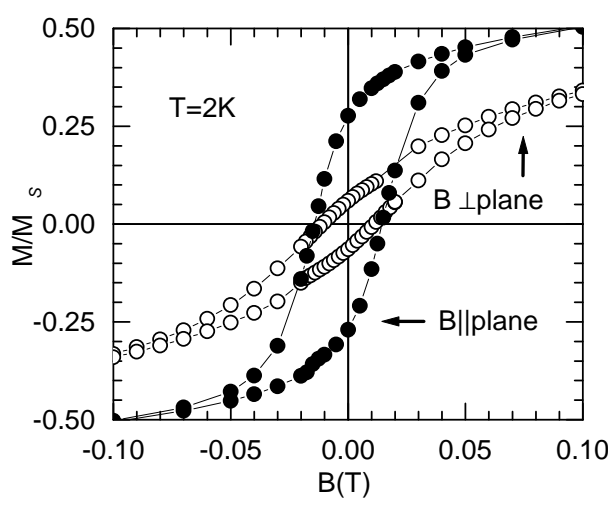

Fig. 3. $\mathrm{M} / \mathrm{M}_{S}$ vs. $\mathrm{B}$ of the Fe-CN-Ni LB film in both orientations. The measurement was made at $2 \mathrm{~K}$.

\section{Discussion and Summary}

A stronger magnetization measured in the parallel orientation in the $\mathrm{M} v s$. T plot in Fig. 2 indicates that the magnetic easy axis is within the XY directions parallel to the film planes. Consistently, in the low field limit $\left(\mathrm{B} \ll \mathrm{B}_{S}\right.$, where $\mathrm{B}_{S}$ is the applied filed at which $\mathrm{M}$ saturates, $\mathrm{M}_{S}$ ), the magnetization in the parallel orientation changes more rapidly than in the perpendicular orientation with respect to changes in the external field. This behavior is most likely due to a combination of the single ion anisotropies of the metal centers and the reduced symmetry of the low-dimensional material. In summary, a 2D Fe-Ni cyanide-bridged network with a face-centered square grid structure has been generated using a Langmuir-Blodgett (LB) synthesis method, and the magnetic study on this film shows the presence of ferromagnetism.

\section{Acknowledgements}

This work was supported, in part, by NSF DMR9900855, NSF DMR-0084173 for the NHMFL, and ACS-PRF 36163-AC5.

\section{References}

[1] J. T. Culp et al., J. Am. Chem. Soc. to appear (2002).

[2] M. Ohba et al., J. Am. Chem. Soc. 119 (1997) 1011.

[3] S. Juszczyk et al., J. Phys.: Condens. Matter 6 (1994) 5697.

[4] O. Kahn et al., J. Am. Chem. Soc. 104 (1982) 2165. 